\title{
Accelerated Corner-Detector Algorithms
}

\author{
Lucas Teixeira, Waldemar Celes and Marcelo Gattass \\ Tecgraf - Computer Science Department, PUC-Rio, Brazil \\ \{lucas, celes, mgattass\}@tecgraf . puc-rio.br
}

\begin{abstract}
Fast corner-detector algorithms are important for achieving real time in different computer vision applications. In this paper, we present new algorithm implementations for corner detection that make use of graphics processing units (GPU) provided by commodity hardware. The programmable capabilities of modern GPUs allow speeding up counterpart CPU algorithms. In the case of corner-detector algorithms, most steps are easily translated from CPU to GPU. However, there are challenges for mapping the feature selection step to the GPU parallel computational model. This paper presents a template for implementing corner-detector algorithms that run entirely on GPU, resulting in significant speed-ups. The proposed template is used to implement the KLT corner detector and the Harris corner detector, and numerical results are presented to demonstrate the algorithms efficiency.
\end{abstract}

\section{Introduction}

In computer vision, different applications can take advantage of fast corner-detector algorithms, such as pattern recognition and multi-view reconstruction. In this paper, the discussion focuses on real-time applications. As a typical example, we can consider tracking techniques, such as Visual SLAM [5], that require an extremely fast corner detector in order to perform all the tasks in a very short period of time. If the goal is to achieve real-time tracking, the whole process has to take less than $33 \mathrm{~ms}$. As a result, the corner-detector phase can only use a fraction of this time.

With the rapid evolution of graphics card technology, GPU-based solutions have outperformed corresponding CPU implementations. High parallel computational capabilities, combined with an increasingly flexible programmable architecture, has turned GPUs into a valuable resource for general-purpose computation. Of special interest in the context of this work, image processing, such as convolution operations and Fast Fourier Transform, can take full advantage of the modern graphics processing power [6].

As a consequence, several studies have been conducted to translate known CPU algorithms to run on GPU. In the case of corner-detector algorithms, most steps are easily translated from CPU to GPU. However, there are challenges for mapping the feature selection step, as defined by Tomasi and Kanade [18] and applied by Shi and Tomasi [15], to the GPU parallel computational model.

This paper presents a template for implementing corner-detector algorithms that run entirely on GPU, resulting in significant speed-ups. The proposed template is used to implement Shi and Tomasi's KLT corner detector [15] and the Harris corner detector [7]. Numerical results are shown to validate the algorithm and to demonstrate its efficiency. 


\section{Related work}

Given an image, the goal of a corner-detector algorithm is to identify the sets of pixels that faultlessly represent the corners in the image. Corner-detector algorithms are usually implemented in two separate steps: corner strength computation and non-maximum suppression. SUSAN [17], Moravec's [12], Trajkovic and Hedle's [19], Shi and Tomasi's [15] and Harris [7] are examples of this class of algorithm. In the corner strength computation step, each pixel is assigned the value of a corner response function, called cornerness value. Generally, this step ends up assigning high values for too many pixels around the vicinity of an image corner. In order to determine only one pixel to represent the position of each corner, the second step selects the pixel with the highest cornerness value in a given neighborhood. In the end, the algorithm ensures that all non-maximum values are suppressed around the considered neighborhood, and thus the pixel that more accurately represents each detected corner is retained. Figure 1 illustrates the conventional steps of corner detection algorithms. In this paper, we are interested in efficient corner-detector algorithms for real-time applications.

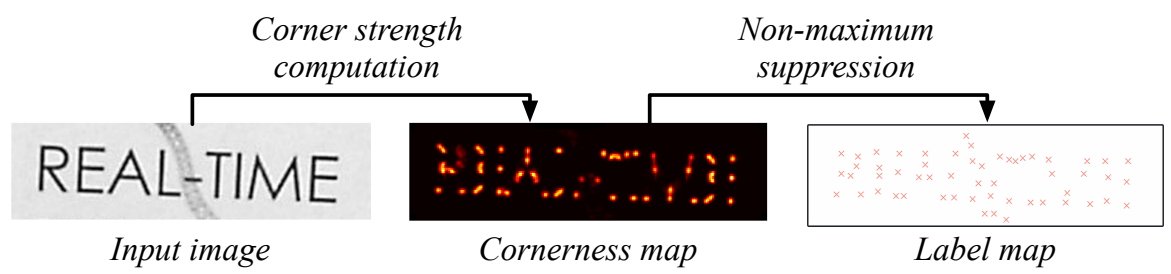

Figure 1: Steps of corner-detector algorithms.

Rosten and Drummond [14] proposed a fast corner-detector algorithm based on machine learning. This algorithm presented good results when applied to Visual SLAM (camera-based simultaneous localization and mapping) applications, but needed to be combined with other algorithms to increase its robustness [9].

Sinha et al.[16] proposed a corner-detector algorithm accelerated by graphics hardware (GPU). However, differently from our proposal, they used the GPU only to compute the "image" with values of a corner response function. To suppress the non-maximum values, the image was read back to be processed on CPU. Although their approach represented a speed-up if compared to the CPU-only counterpart, the gain in performance was not enough for being used in Visual SLAM.

In this paper, we propose an algorithm that is fully implemented on GPU. As a result, our algorithm is able to detect image corners very efficiently, thus being a useful tool for real-time computer-vision applications.

\section{A parallel algorithm for non-maximum suppression}

The computation of an image with corresponding cornerness values associated to the pixels, the cornerness map, can be directly mapped to the GPU parallel model. Once we have the cornerness map, we need to select the pixel with locally maximum values, 
considering a given neighborhood. First, pixels that exceed a given threshold value are retained. This simple procedure reduces the amount of selected pixels, but does not suffice to ensure locally maximums. In order to keep only one pixel per image corner, we have to eliminate previously selected pixels that are within the vicinity of another pixel with greater cornerness value. An approach to perform such an operation on CPU is described by Tomasi and Kanade [18]: the selected pixels are sorted in decreasing order; the pixels are then picked from the top of the list; each pixel is included in the final solution if it does not overlap, within a given neighborhood, with any other pixel already in the solution. Although this is a simple procedure to be implemented on CPU, it cannot be directly mapped to GPU. Therefore, we propose a new parallel algorithm to retain only pixels with locally maximum values.

\subsection{Proposed algorithm}

The algorithms input is a cornerness map where a value equal to zero means that the pixel does not correspond to an image corner. The greater the value, the higher the probability of the pixel being a corner. The algorithms output is another image, named label map, where each pixel is labeled as "inside" or "outside"; the pixels labeled as "inside" belong to the final solution, i.e. represent the position of image corners. During the course of the algorithm, pixels are also labeled as "undefined". In the initialization phase, the pixels with values equal to zero are labeled as "outside", and the others are labeled as "undefined".

The algorithm works in multiple passes. At each pass, all pixels of the image are conceptually processed in parallel, generating an updated label map. The end of each pass represents a synchronization point, and the output of one pass is used as the input of the next pass, together with the cornerness map, to produce a new updated label map. The algorithm thus makes use of two label maps, one for input and another for output. After each pass, the maps are swapped: the output becomes the input and the input becomes the output for the next pass. In the GPU pixel-based parallel computational model, the process associated to each pixel has access to any other pixel from the input map, but can only write to the corresponding pixel in the output map. In other words, gather (indirect read from memory) is supported but scatter (indirect write to memory) is not.

At each pass, the process associated to each pixel accesses corresponding cornerness values, from the cornerness map, and previous labels, from the input label map. If the pixel was previously labeled as "inside" or "outside", the same label is simply copied to the output map. If it is labeled as "undefined", we need to check all the pixels in the neighborhood of the current one. If there exists any other pixel in the neighborhood that is already in the final solution (i.e. labeled as "inside"), the current pixel is discarded from the solution, being labeled as "outside". If no other pixel in the vicinity is labeled as "inside" and the current pixel has the maximum cornerness value among all visited pixels, the current pixel is labeled as "inside". Note that even if there is a pixel in the neighborhood with cornerness value greater than the current pixel, we cannot discard this pixel, because the pixel with greater value may be discarded by another pixel just included in the final solution. As two or more pixels may have the same cornerness value, we have adopted a criterion to disambiguate the order in which the pixels are inserted in the final solution. In the current implementation, we use the pixels $x$ position and then its $y$ position. The algorithm workflow for pixel classification is illustrated in Figure 2. If the 


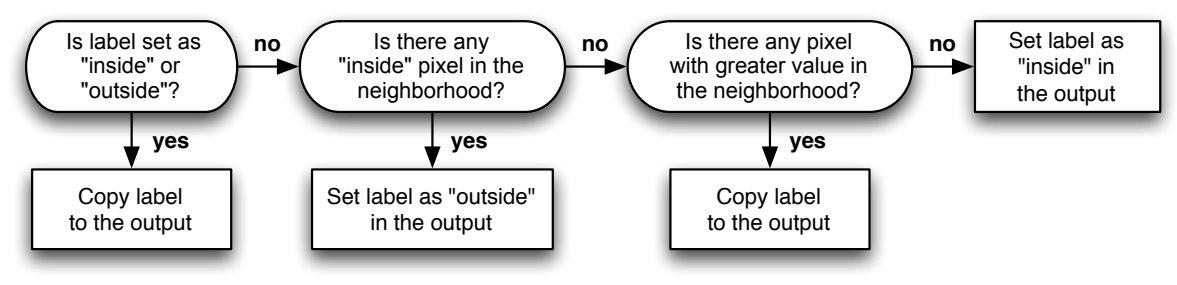

Figure 2: Algorithm workflow for pixel classification.

output map contains pixels labeled as "undefined", a new processing pass is invoked, and the whole process is repeated.

\subsection{Correctness}

In order to demonstrate the correctness of the algorithm, we need to be certified that all pixels are classified correctly and that the algorithm ends. The final solution will be correct if no pixel is wrongly classified as "outside" when supposedly it should be part of the solution, nor wrongly classified as "inside" when it is not part of the solution.

The algorithm described above only classifies a pixel as "inside" if it satisfies two conditions applied to its neighborhood: (i) there is no other pixel that also belongs to the solution; (ii) there is no other pixel with greater cornerness value that can be added to the solution. This ensures that each pixel allowed into the final solution is locally maximal. Conversely, a pixel is only classified as "outside" if another pixel already exists in its neighborhood that is part of the solution (and the latter surely has greater cornerness value).

The algorithm ends when there is no pixel still labeled as "undefined". According to the described algorithm, at each pass at least one pixel is removed from the "undefined" state: the pixel with maximum cornerness value among all 'undefined" pixels is classified as "outside" if there exists one pixel in its neighborhood already in the solution, or as "inside" otherwise. Theoretically, the algorithm may require as many passes as the number of pixels added to the solution, but this is an unreal configuration. In practice, as we shall demonstrate, the algorithm needs only a few passes to converge.

\subsection{Cornerness map compression}

Although GPUs perform memory access very efficiently, the large number of accesses needed to retrieve the information associated to all pixels in the neighborhood of a pixel may degrade performance. In order to reduce the number of memory accesses, we propose a compression of the cornerness map. We can do that by halving the maps dimensions: each $2 \times 2$ box of pixels in the original map is represented by a single pixel in the compressed map. Each compressed pixel then stores the maximum cornerness value among the four original pixels, according to Equation 1 , where $\bar{p}$ denotes the pixels value in the compressed map, $p$ the pixels value in original map, and $(x, y)$ the pixels position.

$$
\bar{p}(x, y)=\max \{p(2 x, 2 y), p(2 x+1,2 y), p(2 x, 2 y+1), p(2 x+1,2 y+1)\}
$$


Together with each cornerness value in the compression map, we also store a flag to indicate which pixel, in the $2 \times 2$ box of the original map, had the maximum value and therefore was selected as the representative pixel in the compressed map. This is important for retrieving the correct pixel position in the original image once the pixel is inserted in the final solution. This compression results in a significant performance gain because it reduces the neighborhood computation by a factor of four. However, it introduces an imprecision of one pixel in the definition of the neighborhood limits.

\subsection{Label map compression}

One common bottleneck in GPU-based image processing algorithms consists of transferring the final resulting image back to the CPU. In order to drastically alleviate this transfer cost, we propose compressing the label map. In the non-maximum suppression step, the algorithm considers a rectangular neighborhood of dimension $\Delta \times \Delta$, where $\Delta$ is an odd number, in order to position the pixel at the center of the neighborhood region. As a consequence, in the final solution, it is guaranteed that in any $f \times f$ region of pixels there is at most only one pixel classified as "inside", where $f$ is given by Equation 2:

$$
f=\frac{\Delta+1}{2}
$$

As a result, we are able to compress the label map dimensions by a factor of $f$, which significantly reduces the amount of memory to be transferred. For example, considering a neighborhood region of $9 \times 9$ pixels, the original map dimensions are reduced by a factor of 5 , thus decreasing the amount of pixels in the label map by a factor of $25\left(=5^{2}\right)$.

A pixel in the compressed label map is labeled as "inside" if there exists one pixel belonging to the corresponding $f \times f$ region in the final solution. For each pixel of the compressed map, the label is stored together with the correct position of the selected pixel in the original image, in order to correctly retrieve the exact corner position; thus, no information is lost.

Combining the compression of both cornerness and label maps, the final label map transferred back to the CPU is reduced by a factor of $(2 f)^{2}$ if compared to the number of pixels in the original image. This is because the dimension of the cornerness map is already reduced by a factor of 2 .

\section{Computational experiments}

The previous section described our proposed template for implementing corner-detector algorithms that run entirely on GPU. In this section, we describe the application of the proposed strategy to two different algorithms: Shi and Tomasis KLT corner detector [15] and the Harris corner detector [7]. Our implementations are compared to previous proposals using different datasets. Quality, performance, and convergence analyses are presented, including the effect of using the proposed map compressions. For reference, the computational experiments were performed on a $2.33 \mathrm{GHz}$ Core 2 Duo processor, 2Gb DD2 RAM, equipped with a NVIDIA GeForce 8800 GTX $768 \mathrm{Mb}$ graphics card, and we used GLSL (the OpenGL Shading Language) [13] for programming the graphics pipeline. 


\subsection{Datasets and implementations}

The computational experiments were executed on three different datasets that are publicly available: (i) Oxford House: a set of 10 images with resolution of $768 \times 576$ pixels [3], illustrated in Figure 3(a). For some experiments, this dataset was resized to other resolutions; (ii) Oxford Corridor: a set of 10 images with resolution of $512 \times 512$ pixels [3], illustrated in Figure 3(b); (iii) Alberta Floor3: a set of 500 images with resolution of $640 \times 480$, captured by manually driving an iRobot Magellan Pro robot around a corridor [8, 10], illustrated in Figure 3(c).

For comparison, we ran the experiments using five different implementations of cornerdetector algorithms. The first two implementations, as listed below, correspond to known third-party implementations. The third implementation was done by the authors following the strategy described by Sinha et al. [16]. The last two implementations are fully GPU-based according to our proposal.

- OpenCV-KLT: a CPU-based optimized implementation of the KLT tracker available in the OpenCV Intel library [2].

- Birchfield-KLT: a CPU-based popular implementation of the KLT tracker by Birchfield [4].

- HALF_GPU-KLT: our implementation of KLT tracker as suggested by Sinha et al. [16]. In this implementation the cornerness map is computed on GPU, but the non-maximum suppression is done using the KLT library by Sinha et al. [16].

- GPU-KLT: our full-GPU implementation of the KLT tracker in accordance with the algorithm proposed by Birchfield [4]. The non-maximum suppression follows our proposed algorithm.

- GPU-HARRIS: our full-GPU implementation of the Harris corner detector. The cornerness map is computed in accordance with Kovesi's code [11], using a $3 \times 3$ Prewitt filter to compute the gradient. The non-maximum suppression follows our proposed algorithm.

In the last two listed implementations, the original image is sent to the GPU, which returns the corresponding computed label map, which can then be processed on the CPU

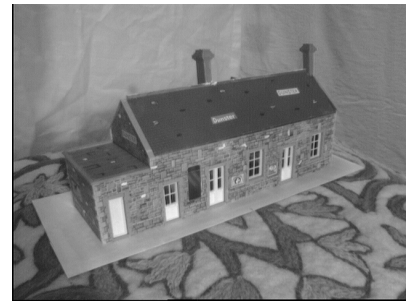

(a) Oxford House

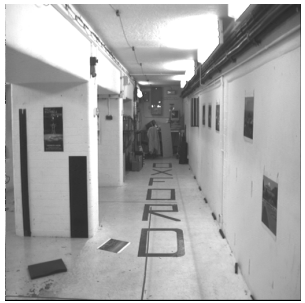

(b) Oxford Corridor

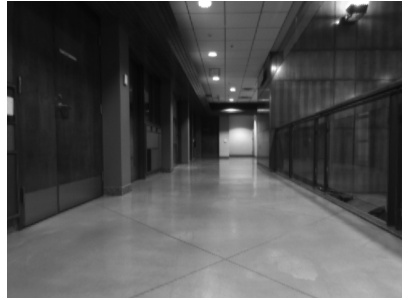

(c) Alberta Floor3

Figure 3: Datasets used in the computational experiments. 
to collect the selected pixels in an array. The dimension of the neighborhood area to compute locally maximum values can vary in the implementations. The GPU-KLT and the GPU-HARRIS implementations present four different variations each: with no compression, with cornerness map compression (CMC), with label map compression (LMC), and with both cornerness map and label map compressions (CMC+LMC).

\subsection{Quality evaluation}

In this section, we compare our GPU-based implementations with corresponding CPUbased counterparts. First, we compare different implementations of the KLT tracker with the Birchfield-KLT implementation. In order to evaluate and compare the implementations, we use the metrics proposed by Klippenstein and Zhang [10]. They proposed plotting recall $/ 1$ - precision curves to evaluate the detectors. The value of recall measures the number of correct matches out of the total number of possible matches, and the value of precision measures the number of incorrect matches out of all matches returned by the algorithms. A perfect detector would produce a vertical line.

This experiment was performed using the Alberta Floor3 dataset, calibrating the methods to extract around 500 features per frame using a neighborhood region of $9 \times 9$ pixels. The goal of the first test was to verify if the difference in hardware (CPU and GPU) can affect the results. For that, we compared the Birchfield-KLT and GPU-KLT implementations, with no compression. As can be seen in Figure 4(a), the corresponding curves are quite similar, with a small difference in precision. We believe this difference is due to the fact that floating point arithmetic in current NVIDIA graphics is very similar to the arithmetic specified by the IEEE 754 standard, but not quite the same [1].

In the second test, we compared the impact of cornerness map compression on the results. Also in Figure 4(a), the GPU-KLT CMC+LMC curve is plotted together with the two previous ones. In Figure 4(b) the same comparison (compressed $v s$. non-compressed) is done for the Harris corner-detector algorithm. As can be noted, this compression can introduce a small difference in precision: around 0.015 for the KLT implementations and around 0.02 for the Harris implementations. This difference is due to the fact that the cornerness map compression introduces an error of 1 pixel in the neighborhood limits.

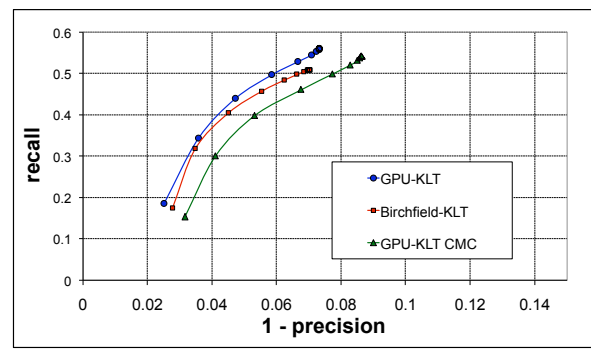

(a) KLT implementations

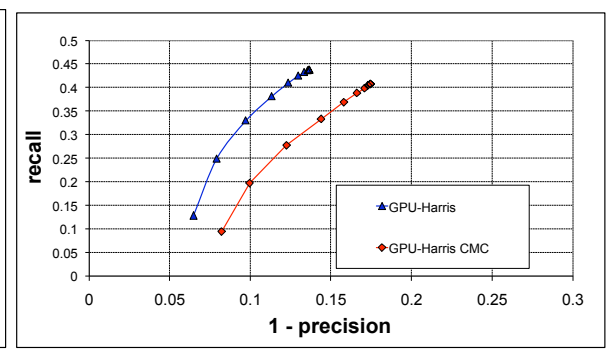

(b) Harris implementations

Figure 4: Comparison using recall/1-precision curves. 


\subsection{Convergence rate}

The main goal of this work is to use GPUs for achieving real-time corner-detector algorithms. Therefore, improving performance is of crucial importance for demonstrating the effectiveness of our proposal. As we are dealing with a multiple-pass algorithm, we have first to check how fast the algorithm converges to the final solution. As described, the algorithm does converge to the final solution but, theoretically, this convergence may be too slow (classifying only one pixel per pass). To verify this, we tested the algorithm's convergence with the three considered datasets.

The test was performed by applying the GPU-HARRIS CMC+LMC algorithm (Harris with both map compressions). We set the neighborhood region to $9 \times 9$ pixels, extracting an average number of 500 features per frame. In order to check the algorithms convergence, we computed the number of detected features per pass. For all three datasets, the first pass sufficed to detect more than $70 \%$ of the total number of features; after three passes, the algorithm had already detected more than $90 \%$ of them. This result demonstrates that the algorithm converges very quickly and suggests that, for real-time applications, one can drastically limit the number of passes, being aware that not all features may be in the final solution, but the majority and most significant of them will. In order to iterate until full convergence is reached, we use occlusion query: the process is stopped when no pixel is added to the final solution in the last iteration.

\subsection{Compression effectiveness}

As shown, the cornerness map compression introduces a small precision error in feature detection because, to compute of locally maximum values, the neighborhood limits vary by +1 pixel. In order to check the effectiveness of both map compressions, we tested the GPU-HARRIS considering all its variations - with and without compressions - using different neighborhood sizes. The Oxford House dataset, reduced to a resolution of $640 \times$ 480 , was used in this test, and an average number of 500 features were extracted per frame.

Table 1 shows the times, in milliseconds, required by each variation of the Harris algorithm. As can be noted, the time spent by the algorithms that do not use cornerness map compression is significantly higher than the ones that compress this map. The reason for this difference in performance is to the large amount of memory accesses needed to retrieve all the information in the neighborhood of an uncompressed pixel. The advantage of compression is even more significant for large neighborhood sizes. These results demonstrate that our proposal makes it possible to use corner-detector algorithms in realtime tracking applications. This test mimics a typical real-time configuration and, with full compression, the algorithm took less than $6 \mathrm{~ms}$ in all the cases.

\begin{tabular}{|c|r|r|r|r|r|}
\hline$\Delta \times \Delta$ & $5 \times 5$ & $9 \times 9$ & $13 \times 13$ & $17 \times 17$ & $21 \times 21$ \\
\hline GPU-HARRIS & 10.1 & 14.0 & 19.0 & 32.0 & 47.0 \\
\hline GPU-HARRIS LMC & 4.4 & 7.3 & 17 & 28.0 & 44.0 \\
\hline GPU-HARRIS CMC & 3.3 & 3.8 & 4.7 & 6.2 & 7.3 \\
\hline GPU-HARRIS CMC+LMC & 2.6 & 2.7 & 3.2 & 4.3 & 5.4 \\
\hline
\end{tabular}

Table 1: Time spent by different variations of the GPU-HARRIS algorithm. 


\subsection{Overall performance}

As a final test, we compared the performance of the proposed fully GPU-based algorithms with the other implementations. Figure 5(a) compares the five implementations for corner detection considered in this work. In this test, both the GPU-HARRIS and the GPU-KTL algorithms were used with full compression. We used the same configuration as in the previous test: Oxford House dataset with different resolutions, extracting an average number of 500 features with neighborhood regions of $9 \times 9$. As can be noted in the figure, the performance of the GPU-accelerated algorithm for both the KLT and the Harris detectors, as proposed here, significantly surpassed previous known implementations. This result clearly demonstrates that our proposal makes it possible to use images with resolution up to $1024 \times 768$ for real-time applications.

To better understand the achieved acceleration of our proposal, Figure 5(b) decomposes the time spent to detect the features at different phases for the algorithms that present best performance. As can be noted, in the CPU implementation the costly phase is the computation of the cornerness map. However, migrating only this phase to the GPU, as proposed by Sinha et al. [16], does not solve the problem because transferring the entire map back to the CPU is also expensive.

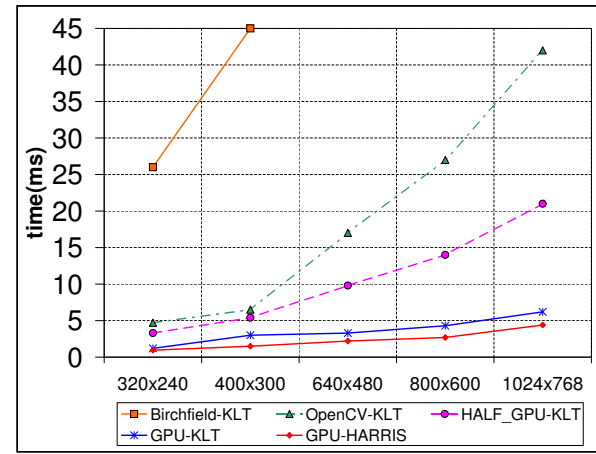

(a) Performace

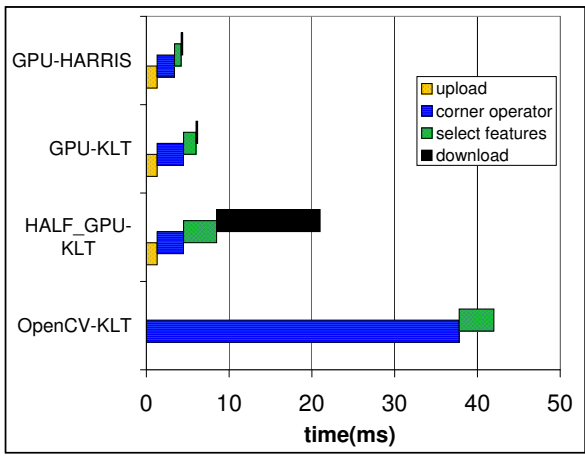

(b) Workload

Figure 5: Comparison among the five considered algorithms.

\section{Conclusion}

In this paper, we presented a new parallel algorithm for non-maximum suppression which allowed the implementation of real-time corner-detector algorithms. The proposed algorithm runs entirely on graphics hardware (GPU) thus eliminating the high cost of memory transferring between the GPU and the CPU. As a result, we were able to implement very efficient KLT and Harris detectors. A set of computational experiments validated the algorithm and demonstrated its efficiency.

In order to achieve real-time processing even for high-resolution images, we propose the use of compressed maps. The compression of the map that stores pixel cornerness values introduced a small precision error, according to the metrics proposed by Klippen- 
stein and Zhang [10]. Nonetheless, map compressions resulted in a significant gain in performance.

We are currently investigating alternative methods for non-lossy cornerness map compressions. One idea is to use the color channels to store all the four values of a $2 \times 2$ region. This will probably impact performance due to the SIMD (single instruction, multiple data) architecture of graphics processors. Further studies have to be carried out in order to better evaluate the benefits.

Acknowledgement. We thank the Oxford Visual Geometry Group for providing access to the Oxford datasets [3]. The Alberta dataset was obtained from the Robotics Data Set Repository (Radish) [8]; thanks go to J. Klippenstein for providing this data.

\section{References}

[1] GPGPU: General-purpose computation using graphics hardware. www . gpgpu . org.

[2] Intel OpenCV: Open source computer vision library. www.intel.com/research/mrl/ research/opencv/.

[3] Oxford datasets. www.robots.ox.ac.uk/ vgg/data/.

[4] S. Birchfield. KLT: An implementation of the Kanade-Lucas-Tomasi feature tracker. www . ces.clemson.edu/stb/klt/, 2007.

[5] A. Davison. Real-time simultaneous localisation and mapping with a single camera. In Proc. International Conference on Computer Vision,Nice, 2003.

[6] O. Fialka and M. Cadik. FFT and convolution performance in image filtering on gpu. Tenth Int. Conf. on Information Visualization, pages 609-614, July 2006.

[7] C. Harris and M. Stephens. A combined corner and edge detector. In Proceedings of The Fourth Alvey Vision Conference, pages 147-152, 1988.

[8] Andrew Howard and Nicholas Roy. The robotics data set repository (Radish), 2003.

[9] Georg Klein and David Murray. Parallel tracking and mapping for small AR workspaces. In Proc. Sixth IEEE and ACM International Symposium on Mixed and Augmented Reality, 2007.

[10] J. Klippenstein and H. Zhang. Quantitative evaluation of feature extractors for visual slam. In Proceedings of the Fourth Canadian Conference on Computer and Robot Vision, 2007.

[11] P. Kovesi. MATLAB and Octave functions for computer vision and image processing. The University of Western Australia. www.csse.uwa.edu.au/ pk/research/matlabfns.

[12] H. Moravec. Obstacle avoidance and navigation in the real world by a seeing robot rover. In tech. report CMU-RI-TR-80-03, Robotics Institute, Carnegie Mellon University. 1980.

[13] R. Rost. OpenGL Shading Language. Addison-Wesley, 2004.

[14] E. Rosten and T. Drummond. Machine learning for high-speed corner detection. In European Conference on Computer Vision, volume 1, pages 430-443, May 2006.

[15] J. Shi and C. Tomasi. Good features to track. In IEEE Conference on Computer Vision and Pattern Recognition (CVPR'94), Seattle, June 1994.

[16] S. Sinha, J. Frahm, M. Pollefeys, and Y. Genc. Feature tracking and matching in video using programmable graphics hardware. Machine Vision and Applications, 2007.

[17] S. Smith and J. Brady. SUSAN - A new approach to low level image processing. Technical Report TR95SMS1c, Chertsey, Surrey, UK, 1995.

[18] C. Tomasi and Kanade T. Detection and tracking of point features. Technical Report CMUCS-91-132, Carnegie Mellon University, 1991.

[19] M. Trajkovic and M. Hedley. Fast corner detection. Image and Vision Computing, 16, 1998. 